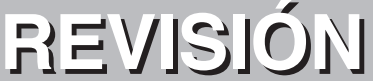

\section{La importancia de los ácidos grasos en la leche materna y en las fórmulas lácteas}

\author{
Por S. Vega, ${ }^{a}$ R. Gutiérrez, ${ }^{a,}{ }^{,}$C. Radilla, ${ }^{b}$ M. Radilla, ${ }^{b}$ A. Ramírez, ${ }^{a}$ J.J. Pérez, ${ }^{a}$ B. Schettino, ${ }^{a}$ \\ M.L. Ramírez, ${ }^{a}$ R. Ortiz ${ }^{a}$ y J. Fontecha ${ }^{c}$ \\ a Profesores Investigadores del Departamento de Producción Agrícola y Animal-Universidad Autónoma \\ Metropolitana - Xochimilco, Calz. Hueso 1100, Col. Villa Quietud, 04960, Coyoacán México, D.F. \\ ${ }^{\mathrm{b}}$ Hospital Médica Sur, Puente de Piedra \#150 Torre 1 Consultorio 430 Col. Toriello Guerra, Tlalpan, \\ CP.14050 \\ ${ }^{c}$ Instituto de Investigación en Ciencia de los Alimentos, Consejo Superior de Investigaciones Científicas, \\ Universidad Autónoma de Madrid, Madrid, España. \\ * Autor para la correspondencia: reygut@correo.xoc.uam.mx
}

\section{RESUMEN}

La importancia de los ácidos grasos en la leche materna y en las fórmulas lácteas.

El alimento natural por excelencia para el recién nacido es la leche materna, cuando no es posible darla se sustituye por fórmulas lácteas, por lo que numerosos estudios enfocan su interés en el análisis de los diversos componentes de éstas, y sus funciones en el lactante. Los ácidos grasos son componentes de gran importancia nutricional tanto en el feto como en el recién nacido. Actualmente se estima que el feto, durante el último tercio del periodo gestacional y el recién nacido, durante los primeros seis meses de vida, requieren un gran aporte de ácidos araquidónico y docosahexaenoico, debido a que la velocidad de transformación de los precursores a nivel hepático no son suficientes para cubrir los requerimientos metabólicos de éstos ácidos grasos y es la madre quien los aporta a través del transporte placentario durante la gestación y a través de la leche durante la lactancia. La Organización para Alimentación y Agricultura (FAO), la Organización Mundial de la Salud (OMS) y la Sociedad Europea de Gastroenterología, Hepatología y Nutrición Pediátrica (ESPGHAN), así como la Academia Americana de Pediatría recomiendan que las fórmulas lácteas deben ser inocuas y similares a la composición de la leche humana y muy especialmente en los ácidos grasos poliinsaturados (PUFA).

PALABRAS CLAVE: Ácidos grasos - Fórmulas lácteas - México.

\section{SUMMARY}

The importance of the fatty acids in breast milk and in lacteal formulae.

The best choice for feeding the newborn child is breast milk, and when it is not possible to breastfeed a baby, the breast milk is replaced by lacteal formula, which is why numerous studies focus their attention on the analysis of the diverse components of baby formula as well as functions in the breastfed baby. Fatty acids are components of great nutritional importance in the fetus and in the newborn child.
Nowadays it is estimated that the fetus, during the last trimester of the gestational period and the newborn child, during the first six months of life, need a great contribution of araquidonic and docosahexaenoic acids, due to the fact that the speed of transformation of the predecessors to the hepatic level are not sufficient to cover the metabolic requirements of these fatty acids and it is the mother who contributes them through placental transport during gestation and her milk during lactation. The Organization of Food and Agriculture (FAO), the World Health Organization (WHO), the European Society of Gastroenterology, Hepatology and Pediatric Nutrition (ESPGHAN) and the American Academy of Pediatrics recommend that lacteal formula imitate the composition of the breast milk, especially in the fatty polyunsaturated acids (PUFA).

KEY-WORDS: Fatty acids - Lacteal formulae - Mexico.

\section{INTRODUCCIÓN}

Las fórmulas lácteas son, hoy por hoy, el sustituto idóneo de la leche humana, también llamada leche materna, cuando ésta no es posible usarla por circunstancias diversas en la alimentación del lactante. Entre los componentes de mayor atención en la leche materna, se encuentran los lípidos y de ellos los que han despertado gran interés en las últimas tres décadas son los ácidos grasos. Se ha demostrado a la luz de la ciencia que los ácidos grasos y, en particular los ácidos grasos insaturados tienen funciones nutracéuticas. Por ejemplo, la deficiencia de ácidos grasos esenciales en la infancia provoca problemas en el desarrollo del cerebro y la retina, además de que previenen y modulan desórdenes autoinmunitarios (neuropatía), cáncer de pecho, cólon y próstata, hipertensión leve, ateroesclerosis, artritis reumatoide, entre otros. Debido a ello, las formulas lácteas que se ofertan en el mercado han sido diseñadas con mezclas de grasas de leches de vaca o cabra con aceites vegeta- 
les y/o animales, como cártamo, girasol, soja y pescado. El objetivo del presente trabajo es dar información actualizada sobre la importancia de los ácidos grasos en la leche materna y en las fórmulas lácteas.

\section{ASPECTOS GENERALES}

Los ácidos grasos poliinsaturados de cadena larga (LC- PUFA, por sus siglas en inglés) han demostrado ser un nutriente esencial en la nutrición humana; tanto en el adulto como en el paciente pediátrico ya que son necesarios para un adecuado crecimiento y funcionamiento corporal (Eritsland, 2000; Rosenfeld et al., 2009). En el recién nacido la leche materna aporta aproximadamente el $50 \%$ de la energía en forma de lípidos, y de éstos el $99 \%$ están en forma de triacilglicéridos (Mena y Milad, 1998; Sanjurjo et al., 2008; Bosch et al., 2009; Martínez et al., 2009). De la misma manera que la madre recibe los ácidos grasos esenciales a partir de la dieta, el feto y el recién nacido los deben recibir de la madre, de ahí que una adecuada y correcta alimentación de la madre es importante para asegurar un buen suministro de todos los nutrientes (Valenzuela y Nieto, 2003; Carmona, 2005; Lama y Moráis, 2005; Adamkin, 2007; Bosch et al., 2009; Campoy et al., 2010; Gil-Campos et al., 2010).

Como se muestra en la Figura 1, es importante destacar el papel de los LC-PUFA específicamente el ácido araquidónico (AA, C20:4 n-6) y el ácido docosahexaenóico (DHA, C22:6 n-3), que mediante procesos de elongación y desaturación son sintetizados en el organismo a partir de los ácidos grasos de 18 átomos de carbono, el linoleico (LA) y el $\alpha$-linolénico (ALA) respectivamente (Haschke-Becher, 2001; Cheatham et al., 2006; Campoy et al., 2010; Valenzuela et al., 2010; Valenzuela et al., 2011).

Mientras, como se esquematiza en la Figura 2, el AA es fácilmente sintetizado del LA (la fuente más abundante de LC-PUFA en la alimentación natural), el DHA experimenta una vía de síntesis más larga y complicada por lo que diversos autores afirman que la tasa de conversión de ALA/DHA es limitada, principalmente en niños nacidos a pretérmino (Mc Namara y Carlson, 2006; Campoy et al., 2010). Es importante hacer énfasis en que los ácidos grasos de cada familia no pueden convertirse entre sí (Morales, 1994; Valenzuela et al., 2011). Por lo tanto, la síntesis de los diferentes ácidos grasos poliinsaturados de cadena muy larga depende directamente de la concentración de los respectivos precursores y es de esencial importancia un aporte correctamente balanceado (Morales, 1994; Valenzuela et al., 2011). La relación ideal entre los ácidos linoleico y $\alpha$-linolénico es de 5:1 a 15:1 (Morales, 1994).

La adición de DHA a las fórmulas lácteas ha supuesto mejoras en las respuestas neurofuncionales y de agudeza visual en los bebés que no son alimentados al seno materno por lo que existen ac- tualmente en el mercado diversas fórmulas lácteas que han incorporado DHA (Simopoulos, 1989; Sala-Vila et al., 2004; Lama y Moráis, 2005; Adamkin, 2007; Prosser et al., 2010; Martínez y Ballew, 2011). Debido a la importancia que recientemente tiene la adición de LC-PUFA, no sólo en las fórmulas lácteas, sino en general en toda la industria alimentaria y farmacéutica, la tecnología busca diversas fuentes de estos ácidos grasos (Sala-Vila et al., 2004; García et al., 2007; Martínez y Ballew, 2011).

Los ácidos grasos n-3 como el ALA existen en varios alimentos naturales como en los aceites de soja y de canola, mientras que el DHA, principalmente se encuentra en pescados, mariscos, algas marinas y actualmente en aceites sintetizados por microorganismos unicelulares (SCO) (Valenzuela y Nieto, 2001; García et al., 2007; Prosser et al., 2010). La variedad de hongos más ampliamente utilizada para la producción de AA comercialmente hablando es la Mortierella alpina, así mismo para la producción de DHA es utilizada el alga marina Crythecodinium cohnii (Sanhueza et al., 2004; García et al., 2007). Algunos preparados para lactantes utilizan LC-PUFA provenientes de aceites de pescado o de los fosfolípidos del huevo y también de AA y DHA sintetizados por microorganismos (Sanhueza et al., 2004). La utilización de aceites SCO incrementa el costo del producto entre un $10-20 \%$ lo que puede retardar la aceptación de estos productos, debido a que el mercado es muy competitivo en precio (García et al., 2007; Martínez y Ballew, 2011).
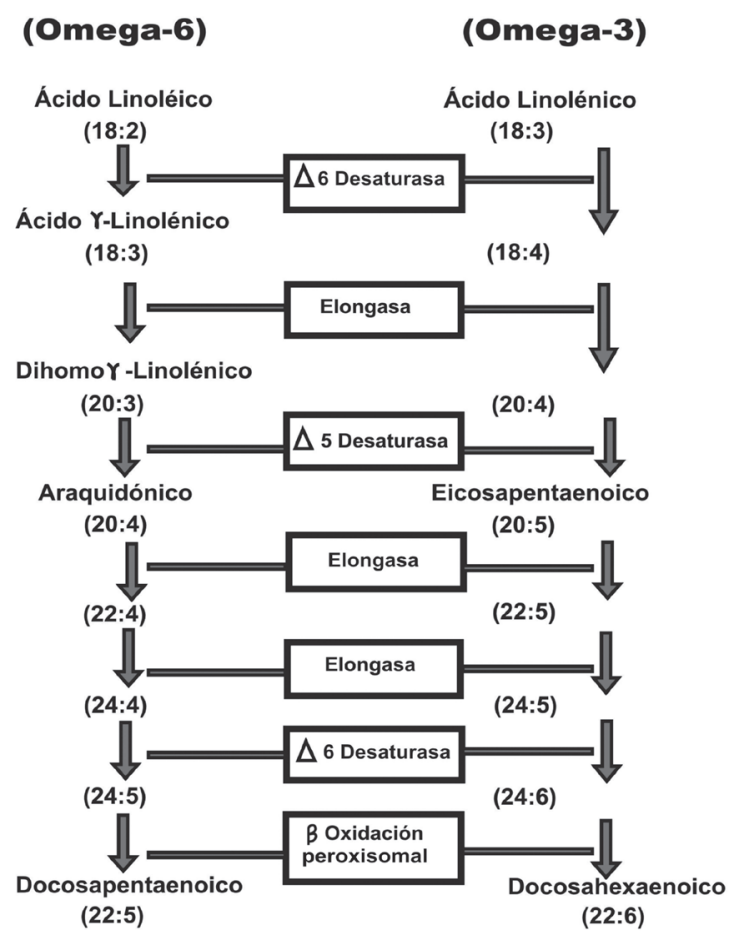

Figura 1

Etapas metabólicas de la biosíntesis de ácidos grasos omega-6 y omega-3 a partir de sus precursores.

Fuente: Modificado de Valenzuela et al., 2011. 


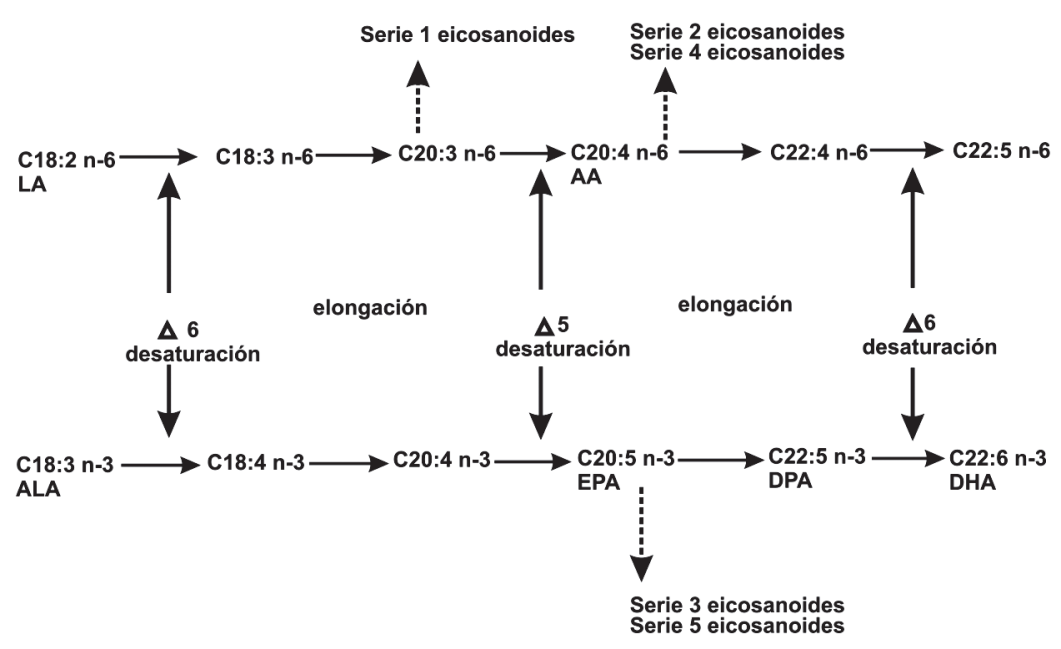

Figura 2

Precursores y síntesis de los ácidos grasos n-3 y n-6.

Fuente: Modificado de Coronado et al., 2006.

\section{IMPORTANCIA FISIOLÓGICA DE LA GRASA}

Las grasas de la dieta se consideran nutrientes de extraordinaria importancia a cualquier edad, pero en los primeros meses de vida, adquieren gran valor por diferentes motivos (Lama y Moráis, 2005), como:

1. Suponen un aporte energético alto sin aumentar la osmolaridad de las fórmulas.

2. Aseguran la utilización proteica óptima y el efecto en la retención nitrogenada es mayor si el aporte energético se realiza con una combinación de hidratos de carbono y lípidos.

3. Los lípidos no polares actúan como aislantes eléctricos que permiten la propagación rápida de las ondas despolarizantes a lo largo de los nervios mielinizados.

4. Facilitan la absorción, el transporte y la disponibilidad de las vitaminas liposolubles.

5. Son un factor importante de saciedad.

La grasa de la dieta que no es oxidada, cerca del $50 \%$ se guarda en los depósitos de reserva energética (tejido adiposo), localizándose en el tejido celular subcutáneo, donde ayuda a mantener la temperatura corporal (aislante térmico) y, en la cavidad abdominal, donde ayuda a proteger órganos vitales como hígado y páncreas. El resto se encuentra cubriendo los vasos (Lama y Moráis, 2005).

Los lípidos son constituyentes importantes de la estructura de las membranas celulares, cumplen funciones energéticas así como de reserva metabólica; también forman la estructura básica de algunas hormonas y de las sales biliares (Valenzuela y Nieto, 2003; Mc Namara y Carlson, 2006; Fernández, 2010; Mourek y Mourek Jr, 2011). Además, algunos lípidos tienen el carácter de esenciales debido a que no pueden ser sintetizados a partir de estructuras precursoras (Valenzuela y Nieto, 2003; Mourek y Mourek Jr, 2011).
Más aún, recientemente se ha identificado la participación de algunos lípidos en la regulación de la expresión génica en los mamíferos, específicamente involucrados en la regulación de los factores de transcripción involucrados en la lipogénesis (Valenzuela y Nieto, 2003; Minihane, 2009). Dentro de la gran diversidad estructural que caracteriza a los lípidos, los ácidos grasos son quizás las estructuras de mayor relevancia (Valenzuela y Nieto, 2003).

En cuanto a la función de los ácidos grasos y su funcionamiento sobre los cambios del desarrollo del organismo, se resalta lo siguiente:

1. En el tejido de la corteza cerebral la parte de DHA en la edad adulta representa aproximadamente el $20 \%$ y la parte de AA es de aproximadamente el $10 \%$ de todos los ácidos grasos detectados. Filogenéticamente, en la partes más antiguas del cerebro la parte de DHA es más pequeña.

2. Existe una relación significativa entre el peso del recién nacido al momento del nacimiento y la concentración de PUFA n-3 en sangre.

3. Cuando el organismo presenta desnutrición la concentración de PUFA n-3 en el suero sanguíneo disminuye. Con ello, las concentraciones de ácidos grasos saturados se elevan rápidamente sobre todo en aquellos que tienen una cadena más corta.

4. Las etapas iniciales del desarrollo de organismo en los mamíferos se caracterizan por una concentración más alta de ácidos saturados grasos (Mourek y Mourek Jr, 2011).

Hallazgos recientes evidencian que la concentración intestinal de la lipasa triacilglicérido-pancreática $(P T L)$ y sales biliares es inferior en recién nacidos en comparación con etapas ulteriores en la vida. En cambio, la proteína relacionada PTL-2 y la lipasa estimulada por sales biliares (BSSL) son las enzimas claves secretadas del páncreas, las cuales en conjunto con la acción de la lipasa funcionan 
para alcanzar la absorción eficiente de las grasas durante la primera infancia. La BSSL está también presente en la leche materna, entre sus funciones, modifica la absorción de grasas y el crecimiento en recién nacidos prematuros. En condiciones de bajas cantidades de sales biliares a nivel luminal, las concentraciones de absorción de las grasas probablemente ocurren en las fases de producto líquidas cristalinas, que pueden causar la absorción en una amplia parte de la superficie mucosa del intestino delgado en comparación con los adultos. El ensamble y secreción de los quilomicrones también parece adaptarse a la situación específica del recién nacido; por ejemplo, en pretérmino tienden a malabsorción lipídica debido a la baja actividad de la lipasa pancreática y a la insuficiente formación de sales biliares (Lindquist y Hernell, 2010).

En los últimos años se ha comprendido más ampliamente los efectos de las grasas poliinsaturadas (PUFA) en la regulación de factores de transcripción involucrados en la lipogénesis y metabolismo de las lipoproteínas, se ha evidenciado mayor efecto de ácidos grasos omega 3, especialmente EPA y DHA que los ácidos grasos omega 6 (Minihane, 2009).

\section{IMPORTANCIA DE LOS ÁCIDOS GRASOS EN LAS PRIMERAS ETAPAS DE LA VIDA}

\subsection{Etapas gestacional y lactancia}

La ingesta de lípidos en la gestación y durante el primer año de vida del humano es fundamental, no sólo para cubrir las necesidades energéticas, sino también como vehículo de las vitaminas liposolubles para favorecer la absorción de éstas y como fuente importante de LC-PUFA. Durante la etapa gestacional, e incluso después del nacimiento, el aporte de LC-PUFA es realizado por la madre, ya que si bien el feto y el recién nacido tienen la capacidad para formar LC-PUFA a partir de sus precursores, la velocidad de transformación (elongación y desaturación) del LA para formar AA y del ALA para formar DHA, parece no ser suficiente para proveer la cantidad de LC-PUFA requeridos (Simopoulos, 1989; Koletzko et al., 2001; Valenzuela y Nieto, 2003; Cunninghaim y Mc Dermott, 2008; GilCampos y Dalmau, 2010).

En el embarazo se produce toda una serie de cambios importantes en el metabolismo con el objeto de lograr un aporte adecuado de nutrientes desde la madre hacia el feto, además de incrementar los depósitos de lípidos maternos durante los primeros meses de la gestación para cubrir las necesidades de energía al final del embarazo y durante la lactancia (Mc Gregor et al., 2003; Campoy et al., 2010). Durante la primera mitad de la gestación existe una fase anabólica que se caracteriza por un aumento de la capacidad materna para almacenar proteínas y energía en forma de grasa, cuando las necesidades fetales son aún relativamente pequeñas (Mc Gregor et al., 2003; Innis, 2007; Campoy et al., 2010). La fase catabólica ocurre durante la segunda mitad de la gestación, durante este periodo la madre utiliza como combustible energético lípidos en lugar de glucosa (Mc Gregor et al., 2003; Innis, 2007; Campoy et al., 2010).

A lo largo del embarazo, el feto acumula los elementos requeridos para el crecimiento, oxida los sustratos energéticos transportados desde la madre y elimina productos metabólicos hacia la madre, y en la última mitad de la gestación, también acumula depósitos de energía para cubrir las necesidades neonatales (Mc Gregor et al., 2003; Campoy et al., 2010).

La dieta fetal está constituida por una elevada cantidad de hidratos de carbono, cantidades adecuadas de sustancias nitrogenadas y muy baja cantidad de grasas, con la excepción de los ácidos grasos poliinsaturados, que últimamente están recibiendo mucho interés científico debido a las funciones que tienen éstos en el feto (Mc Gregor et al., 2003; Innis, 2007; Campoy et al., 2010). En el metabolismo de los lípidos del feto, se incluyen la incorporación de ácidos grasos estructurales como fosfolípidos, la esterificación en triacilglicéridos, el depósito en tejido adiposo y su oxidación; pero la actividad biosintética de elongación y desaturación del hígado fetal es muy incipiente debido a la inmadurez fisiológica de éste órgano (Koletzko et al., 2001; Valenzuela y Nieto, 2003; Campoy et al., 2010). Los ácidos grasos esenciales LA y ALA y sus derivados de cadena larga, AA y DHA deben ser aportados desde la circulación materna a través de la placenta, para la formación de triacilglicéridos y fosfolípidos, ya que la placenta humana no tiene la capacidad para elongar y desaturar los LCPUFA precursores, sin embargo es selectivamente permeable a los PUFA de origen materno (Carmona, 2005; Campoy et al., 2010). De la misma forma que la madre recibe los ácidos grasos esenciales a partir de la dieta, el feto los debe recibir de las reservas tisulares de PUFA de la madre (principalmente del tejido adiposo), de la actividad biosintética (elongación y desaturación de precursores) y del aporte nutricional adecuado de PUFA (Figura 3) (Carmona, 2005; Bosch et al., 2009; Campoy et al., 2010). Por eso es importante la buena alimentación de la madre, para asegurar el suministro de todos los nutrientes que el feto requiere (Carmona, 2005; Cheatham et al., 2006; Contreras et al., 2011; Makrides et al., 2011).

Los LC-PUFA n-3 y n-6 son básicos para el desarrollo cerebral fetal y cognoscitivo del recién nacido, ya que se encuentran en más del $30 \%$ de los ácidos grasos que forman los fosfolípidos que integran las membranas celulares del sistema nervioso. Desde la placenta son utilizados para el desarrollo del cerebro, para su incorporación a los lípidos estructurales de los tejidos, para su almacenamiento en forma de triacilglicéridos en el tejido adiposo, especialmente durante el último trimestre de la gestación y para su utilización como fuente de energía (Campoy et al., 2010; Makrides et al., 2011). 


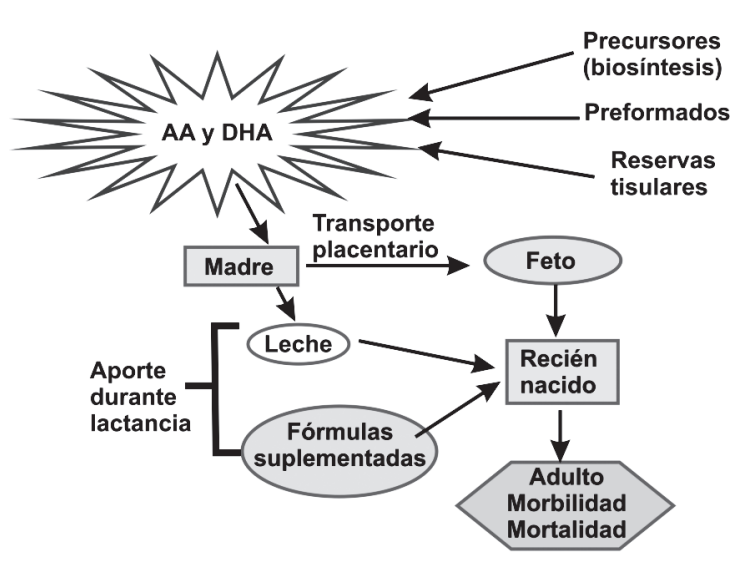

Figura 3

Aporte de ácidos grasos omega-3 durante las etapas gestacional y lactancia.

Fuente: Modificado de Valenzuela et al., 2003.

Sobre el último trimestre del embarazo, hay un $35 \%$ de incremento en el nivel del DHA en la retina $\mathrm{y}$, los recién nacidos pretérmino es posible que sean más susceptibles de cualquier reducción en la disponibilidad del DHA para acumularlo en esta zona. Los bastones de la retina tienen más del 50\% de los ácidos grasos de la familia n-3, principalmente DHA. Las membranas de estas células contienen pigmentos fotosensibles que absorben la luz e inician la excitación visual, esto genera señales eléctricas que son transmitidas a la corteza occipital en milisegundos. Estos cambios rápidos requieren la presencia de $\mathrm{DHA}$, ya que se han observado cambios en la función de la retina en ratas alimentadas con una dieta deficiente en ácidos grasos $\mathrm{n}-3$, presentando una disminución en las señales eléctricas generadas por la luz (Rodríguez et al., 2004; Campoy et al., 2010; Gil-Campos, 2010).

La importancia de la adecuada ingestión de lípidos durante las edades infantiles es tanto cualitativa como cuantitativa. La mitad de la energía que requiere un recién nacido para el crecimiento y mantenimiento de las funciones sistémicas y corporales es proporcionada mediante el contenido graso de la leche materna. Es importante conocer que la dieta materna, la tasa de transferencia placentaria, y el aporte exógeno por medio de la leche humana son algunos de los factores que controlan el estado lipídico y la biodisponibilidad metabólica y nutricional de los LC-PUFA en el recién nacido. La estrategia intervencionista estará entonces encaminada a apoyar la dieta de la gestante, mantener el estado de salud en todas las facetas de la futura madre; y mantener la lactancia materna exclusiva durante los primeros 4-6 meses de vida del niño (Novo, 2010).

\section{LÍPIDOS EN LECHES HUMANA, VACA Y FÓRMULAS LÁCTEAS}

\subsection{Composición de las leches humana y vaca}

En la leche humana existen más de 200 componentes reconocidos. Durante los siete primeros días postparto, a la leche producida se le denomina calostro, el cual destaca por su alto contenido en proteínas como factores inmunológicos, llegando tener hasta $23 \mathrm{~g} / \mathrm{L}$ de proteínas totales; 1400 mg/L de caseína; 2180 mg/L de alfa lactoalbúmina; $3300 \mathrm{mg} / \mathrm{L}$ de lactoferrina y 3640 mg/L de lgA (Vásquez, 2005; Macías et al., 2006; García, 2011). Después de la primera semana, la leche va cambiando su composición y dos a tres semanas después tiene las características de la leche madura (Mena y Milad, 1998; Vásquez, 2005; Macías et al., 2006; García, 2011). A la luz de la ciencia se ha demostrado que la composición de la leche humana varía según la edad de gestación (Vásquez, 2005; Macías et al., 2006; García, 2011; Valenzuela et al., 2011). La leche prematura tiene mayor cantidad de proteínas $(23 \mathrm{~g} / \mathrm{L})$ y menor cantidad de lactosa $(55 \mathrm{~g} / \mathrm{L})$ que la leche madura $(10 \mathrm{~g} / \mathrm{L}$ proteínas y $73 \mathrm{~g} / \mathrm{L}$ lactosa), como si se adaptara a las condiciones fisiológicas del recién nacido (Vásquez, 2005; Macías et al., 2006). No obstante, se sabe que los niños prematuros alimentados por su propia madre, requieren para alcanzar una velocidad de crecimiento semejante a la intrauterina, de suplementos de proteínas, minerales y algunos oligoelementos (Innis, 2003; Macías et al., 2006).

La cantidad de lípidos contenidos en la leche humana es de alrededor de $35-45 \mathrm{~g} / \mathrm{L}$, y constituyen la mayor fuente energética de la misma (40-55\%) (Macías et al., 2006; García, 2011). Son transportados dentro del glóbulo de grasa cuya membrana está compuesta principalmente de proteínas, fosfolípidos y colesterol (100-150 mg/L), en tanto que el interior del glóbulo de grasa lo constituyen principalmente triacilgilcéridos (Macías et al., 2006; García, 2011). El contenido de grasa se aproxima al $98 \%$ de triacilglicéridos y $2 \%$ de colesterol, ésteres de colesterol y fosfolípidos (Macías et al., 2006; Ros-Berruezo, 2007; García, 2011). Además el contenido en ácidos grasos poliinsaturados de cadena larga como el ácido araquidónico (AA) y el docosahexaenóico (DHA) es mayor en la leche humana que en la leche de vaca (Macías et al., 2006; García, 2011; Valenzuela et al., 2011).

La leche humana contiene ácidos grasos poliinsaturados de cadena larga, destacando el contenido de los ácidos araquidónico $(0.44 \mathrm{~g} / 100 \mathrm{~g}$ de gra$\mathrm{sa})$, docosahexaenoico (0.30 $\mathrm{g} / 100 \mathrm{~g}$ de grasa) y $\gamma$-linolénico (0.12 g/100g de grasa) (Morales, 1994).

La digestión y la absorción de los ácidos grasos procedentes de la grasa de la leche de vaca es menor que la de los lípidos de la leche humana debido a que la actividad de la lipasa intestinal produce 1,3-monoacilgliceroles en vez de 2-monoacilgliceroles, en particular, ácido palmítico, que son las formas que se absorben con mayor facilidad en el intestino de los lactantes (Macías et al., 2006). Los lípidos de la leche humana varían ampliamente con la etapa de la lactancia, su concentración es mayor al final que al inicio, tiene ritmo diurno, puede ser diferente entre una y otra glándula y varía de un individuo a otro (Mena y Milad, 1998). Parece ser que la concentración de lípidos en la leche humana 
Tabla 1

Composición representativa de la leche de vaca y la leche humana madura

\begin{tabular}{|c|c|c|}
\hline Componente & $\begin{array}{l}\text { Leche humana } \\
\text { madura }\end{array}$ & Leche de vaca \\
\hline Calorías (cal/L) & 750 & 701 \\
\hline Minerales cationes (mEq/L) sodio, potasio, calcio, magnesio & 50 & 150 \\
\hline Minerales aniones (mEq/L) fósforo, azufre, cloro & 40 & 110 \\
\hline \multicolumn{3}{|l|}{ Oligoelementos (mcg/dL) } \\
\hline Hierro & $3 \mathrm{mg} / \mathrm{dL}$ & $46 \mathrm{mcg} / \mathrm{dL}$ \\
\hline Cobre & 1.1 & 10 \\
\hline Zinc & 30 & - \\
\hline Proteínas (g/L) & 23 & 32 \\
\hline Aminoácidos (g/L) & 12.8 & 32 \\
\hline Nitrógeno no proteico (mg/L) & $30-500$ & 252 \\
\hline Lisozima (mg/L) & 390 & 0.13 \\
\hline Hidratos de carbono (g/L) & $60-70$ & 47 \\
\hline Grasas (g/L) & $35-45$ & 38 \\
\hline \multicolumn{3}{|l|}{ Vitaminas (mg/L) } \\
\hline Vitamina A & 0.61 & 0.27 \\
\hline Caroteno & 0.25 & 0.37 \\
\hline Tocoferol & 2.4 & 0.6 \\
\hline Tiamina & 0.142 & 0.43 \\
\hline Riboflavina & 0.373 & 1.56 \\
\hline Vitamina $\mathrm{B}_{6}$ & 0.15 & 0.51 \\
\hline Ácido nicotínico & 1.83 & 0.74 \\
\hline Vitamina $\mathrm{B}_{12}(\mathrm{mcg} / \mathrm{L})$ & 0.5 & 6.6 \\
\hline Biotina (mcg/L) & 2 & 22 \\
\hline Ácido fólico & $24-30 \mathrm{mg} / \mathrm{L}$ & $35-40 \mathrm{mg} / \mathrm{L}$ \\
\hline Ácido pantoténico & 2.5 & $3-4$ \\
\hline Ácido ascórbico & 52 & 10 \\
\hline
\end{tabular}

Fuente: Modificado de García, 2011.

está asociada al tipo de lípidos ingeridos por la madre y con la conformación de lípidos de sus reservas en el tejido adiposo (Mena y Milad, 1998; Macías et al., 2006; García, 2011; Valenzuela et al., 2011). Además, cuando la dieta es pobre y las reservas escasas, la cantidad que contiene la leche disminuye como sucede en mujeres con alimentación deficiente (Mena y Milad, 1998; Macías et al., 2006; García, 2011; Valenzuela et al., 2011).

Se requieren diversas modificaciones para obtener, a partir de la leche de vaca, una fórmula para lactantes cuya composición sea similar a la de la leche humana.

\subsection{Fórmulas lácteas}

Durante el siglo pasado, el recurso de la alimentación láctea con biberón, u otros utensilios despertó el interés entre la población (Sánchez et al., 2008). Sin embargo, desde entonces fue reconocido el riesgo de infección del lactante alimentado con este método (Sánchez et al., 2008). Forsyth en
1910 pensaba que la leche de vaca era una opción de la leche humana, y que el pecho materno no era esencial para el lactante (Mepham, 1993; Sánchez et al., 2008). Desde entonces, se han visto muchos cambios en la alimentación del lactante, desde la mejora extraordinaria de las leches industrializadas y fórmulas modificadas, pasando por las grandes controversias sobre su uso en población con condiciones higiénicas deplorables, hasta el resurgimiento a partir de la década de los setenta de la alimentación con leche materna, que en la actualidad se considera insuperable por la gran cantidad de propiedades nutritivas, inmunológicas y psicoafectivas que hasta la fecha no han logrado las fórmulas lácteas, ya que ha sido un verdadero reto para los productores de fórmulas infantiles diseñar y fabricar una fórmula que se acerque a los estándares de referencia de la alimentación infantil (Morris et al., 2000; Carver, 2003; Gil et al., 2006; Sánchez et al., 2008; Prosser et al., 2010; Martinez y Ballew, 2011).

Como ya se comentó, la leche materna asegura un aporte y una relación adecuada de PUFA ome- 
ga-6 y omega-3 (Valenzuela y Nieto, 2003; Valenzuela et al., 2011). Sin embargo, este aporte se puede ver modificado si el periodo de lactancia es menor y/o alterado, o si simplemente este no es posible (Valenzuela y Nieto, 2003; Valenzuela et al., 2011). Las fórmulas lácteas han ido incorporando, en la medida que es tecnológicamente posible, los componentes fundamentales de la leche materna y aunque aún su composición dista mucho para igualarse a la secreción láctea, en los últimos años se han logrado progresos muy importantes (Carver, 2003; Valenzuela y Nieto, 2003; Prosser et al., 2010; Martinez y Ballew, 2011). En lo que respecta al tipo de ácidos grasos que aportan estas fórmulas, es necesario identificar aquellas que contienen LA y ALA y aquellas que además contienen AA y DHA, ya que la tendencia actual es adicionar los alimentos infantiles con estos ácidos grasos; y el problema surge cuando el lactante debe alimentarse con fórmulas convencionales, generalmente ricas en ácido linoleico, en algunos casos con aportes suficientes de ácido $\alpha$-linolénico, pero pobres en ácido docosahexaenoico y araquidónico (Morales, 1994; Surh et al., 2007; Rosenfeld et al., 2009; Prosser et al., 2010; Makrides et al., 2011; Martinez y Ballew, 2011).

Considerando los antecedentes clínicos y experimentales que demuestran que el recién nacido no tendría una capacidad totalmente desarrollada para realizar los procesos de elongación y desaturación de los precursores LA y ALA, parece más lógico y más seguro desde el punto de vista de biodisponibilidad de PUFA, aportar AA y DHA en las fórmulas, además del LA y ALA (Valenzuela y Nieto, 2003; Martinez y Ballew, 2011). De esta manera, es posible asemejar mejor el perfil de ácidos grasos de la fórmula con el de la leche humana (Valenzuela y Nieto, 2003; Martinez y Ballew, 2011).

La composición y el uso de los sustitutos de la leche materna sólo han empezado a regularse y armonizarse a niveles nacionales e internacionales en los últimos 30 años (Sala-Vila, 2004; Paulou et al., 2010). En la Unión Europea la composición de las fórmulas para lactantes, y de las fórmulas de continuación, están reguladas por Directivas y Enmiendas elaboradas desde 1991 (Vásquez, 2005; Vásquez-Garibay y Romero-Velarde, 2008). La Directiva de 1991 permitía a los Estados Miembros de la Unión Europea presentar propuestas de enmienda a la composición de las fórmulas para lactantes y a partir del mismo año, se autorizó la inclusión de nucleótidos, selenio y ácidos grasos de cadena larga (Vásquez, 2005; Vásquez-Garibay y Romero-Velarde, 2008). La Unión Europea regula más de 50 componentes de las fórmulas lácteas y establece los límites superiores para la mayoría de éstos, lo que supone una normativa más rigurosa que la de la "Food and Drugs Administration" (FDA) de Estados Unidos (Vásquez, 2005; Vásquez-Garibay y Romero-Velarde, 2008; Paulou et al., 2010).

La leche humana es un complejo y completo alimento, específico para el recién nacido, que en condiciones normales le provee de todos los nu- trientes requeridos para el perfecto desarrollo durante los primeros 4-6 meses de vida, y por tanto, debe constituir el método de elección de alimentación del lactante (Vásquez, 2005; Vásquez-Garibay y Romero-Velarde, 2008; García, 2011). No obstante en ocasiones se puede contraindicar la lactancia materna, por ejemplo cuando la madre está bajo tratamiento de algún padecimiento y se le están suministrando fármacos que son transferidos al lactante o cuando se presenta intolerancia a la leche. En este caso se ha de recurrir a las fórmulas lácteas (Vásquez, 2005). Éstas han sido diseñadas y reguladas por diferentes comités, para asegurar que las necesidades nutricionales de los lactantes sean cubiertas (Vásquez, 2005).

La Unión Europea (2006/141/CE) ampara las siguientes definiciones (Vásquez, 2005; VásquezGaribay y Romero-Velarde, 2008):

Fórmulas lácteas de inicio: Son productos alimenticios destinados a la alimentación especial de los lactantes durante los primeros meses de vida que satisfagan por sí mismos las necesidades nutritivas de estos lactantes hasta la introducción de una alimentación complementaria apropiada.

Fórmulas de continuación: Son productos alimenticios destinados a la alimentación especial de los lactantes, cuando se introduce una alimentación complementaria apropiada y constituye el principal elemento líquido de una dieta progresivamente diversificada en estos lactantes.

La composición de las grasas en la Fórmula de Inicio debe ser tal que consiga una absorción del 85\% (Sánchez et al., 2008). Se permite una mezcla de grasas animales y vegetales para conseguir un ácidograma similar al de la leche materna (Sánchez et al., 2008). En cuanto a las grasas saturadas, su concentración debe ser pequeña, mientras que la de los ácidos grasos monoinsaturados debe ser más elevada (Sánchez et al., 2008). En la Fórmula de Continuación no hay razón para sustituir totalmente la grasa animal por la vegetal, pero cuando la fórmula contiene grasas vegetales, el contenido de ácido linoleico es inferior a 300mg/ Kcal (Sánchez et al., 2008).

En España durante la Conferencia de Consenso sobre Lípidos en Pediatría (1998) se señaló que para cubrir el $30-35 \%$ de la energía total en forma de grasa, la distribución de los ácidos grasos debe ser la siguiente: $10 \%$ del total como aporte de ácidos grasos saturados; $10-20 \%$ como ácidos grasos monoinsaturados y 7-10\% como ácidos grasos poliinsaturados, con una relación $n-6 / n-3$ comprendida entre 5 y 15 (Gil et al., 2006). La Tabla 2 indica las recomendaciones de varios organismos internacionales de ingesta de grasa para los lactantes y niños de corta edad.

\section{EL PAPEL DE LOS LC-PUFA EN EL DESARROLLO VISUAL Y CEREBRAL}

Evidentemente existe una relación directa entre una buena nutrición y un desarrollo infantil óptimo, 
Tabla 2

Recomendaciones de ingesta de grasa, expresada como porcentaje de la energía total, para lactantes y niños de corta edad

\begin{tabular}{|c|c|c|c|c|}
\hline & \multicolumn{4}{|c|}{ Intervalo de edad } \\
\hline & $0-4 / 6$ meses & 6-12 meses & $12-24$ meses & 24-36 meses \\
\hline $\begin{array}{l}\text { Academia Americana de Pediatría. Comité de } \\
\text { Nutrición, } 1986\end{array}$ & & & & $30-40$ \\
\hline Academia Americana de Pediatría,1992 & & & & 30 \\
\hline $\begin{array}{l}\text { Sociedad Europea de Gastroenterología, } \\
\text { Hepatología y Nutrición Pediátrica (ESPGHAN } \\
\text { 1991,1994, 2005) }\end{array}$ & $\geq 40-58.5$ & $\geq 32-58.5$ & Sin restricción & $30-35$ \\
\hline WHO/FAO, 1994 & $50-60$ & & $30-40$ & $30-40$ \\
\hline Unión Europea, 1996 & $\geq 40-58.5$ & $\geq 32-58.5$ & & \\
\hline $\begin{array}{l}\text { Instituto de Medicina. Academia de Ciencias de } \\
\text { Estados Unidos, } 2002\end{array}$ & 60 (31g/día) & 55 (30 g/día) & & \\
\hline
\end{tabular}

Fuente: Modificado de Uauy y Dangour, 2009.

particularmente en el cerebro (Colombo et al., 2004; Georgieff, 2007; Campoy et al., 2010). El desarrollo del sistema nervioso, ocurre en el último tercio del periodo gestacional, en el caso del humano, durante los últimos 3 meses del embarazo (Valenzuela y Nieto, 2001; Makrides et al., 2011). Sin embargo algunos estudios han informado que el desarrollo cerebral es capaz en cierta medida resistente a las deficiencias nutricionales (Cheatham et al., 2006; Ros-Berruezo, 2007), aunque no completamente, ya que es aquí donde comienza en forma activa la formación de las neuronas y donde el requerimiento de DHA aumenta considerablemente (Valenzuela y Nieto, 2001; Ros-Berruezo, 2007; Makrides et al., 2011). La Figura 4, muestra un modelo hipotético del metabolismo de los ácidos grasos omega-6 y omega-3 en el cerebro.

EI DHA es un componente esencial en el sistema nervioso y las altas concentraciones en el córtex y la retina indican su importancia en el desarrollo y mantenimiento de la función neural y visual, donde regula funciones de la membrana como canales de iones, actividades de receptor y actividades enzimáticas proporcionando ultraestructuras específicas dentro

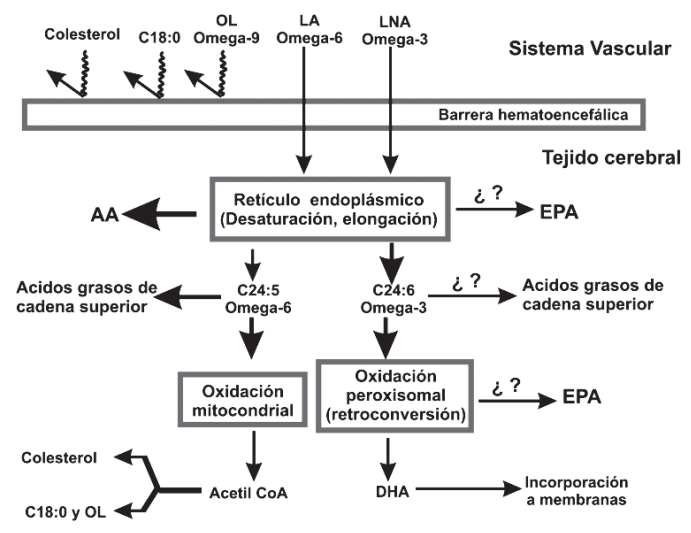

Figura 4

Metabolismo de ácidos grasos omega-6 y omega-3 en el cerebro.

Fuente: Modificado de Coronado et al., 2006. de la bicapa fosfolipídica (Valenzuela y Nieto, 2001; Auestad et al., 2003; Colombo et al., 2004; Makrides et al., 2011; Mourek y Mourek Jr, 2011).

El contenido de DHA a nivel cerebral es del 12$15 \%$ y se acumula principalmente en los fosfolípidos cerebrales, particularmente en la fosfatidilcolina y la fosfatidiletanolamina, y en menor proporción en la fosfatidilserina, inositol y en una gran variedad de plasmalógenos (esfingolípidos) (Valenzuela y Nieto, 2001; Sanhueza et al., 2004; Whelan, 2008; Makrides et al., 2011; Mourek y Mourek Jr, 2011). El porcentaje de triacilglicéridos del tejido cerebral es muy bajo en comparación a los fosfolípidos, por lo cual el contenido total de DHA en los primeros es muy pequeño (Valenzuela y Nieto, 2001; Cheatham et al., 2006; Makrides et al., 2011; Mourek y Mourek Jr, 2011). El DHA se acumula principalmente en la posición sn-2 de los fosfolípidos, esto es esterifica al hidroxilo central del glicerol que forma parte de los fosfolípidos (Valenzuela y Nieto, 2001; Sanhueza et al., 2004). La posición sn-1 está casi siempre ocupada por un ácido graso saturado, principalmente palmítico (C16:0) (Valenzuela y Nieto, 2001; Sanhueza et al., 2004). El AA comparte la misma posición sn-2 que el DHA en los fosfolípidos (Sanhueza et al., 2004; Mourek y Mourek Jr, 2011).

Los campos del comportamiento pueden en general ser categorizados como sensoriales, motores, cognoscitivos y sociales (Cheatham et al., 2006).Las diferencias en éstos ocurren debido a cambios en la estructura y la función cerebral (Valenzuela y Nieto, 2001; Cheatham et al., 2006). El DHA y AA son los mayores componentes estructurales en el cerebro que decrecen cuando se consumen dietas deficientes en ácidos grasos esenciales ALA y LA (Georgieff, 2007; Campoy et al., 2010). De acuerdo a algunos estudios, al parecer los bebés a término son perfectamente capaces de sintetizar DHA de la serie n-3 y AA de la n-6 en cantidades suficientes para su desarrollo cerebral normal a partir de dietas que contengan los precursores de las dos series en proporciones adecuadas (Sanhueza et al., 2004; Campoy et al., 2010). 
Tabla 3

Velocidad de incorporación de ácidos grasos en el cerebro y el cerebelo fetal ( $\mathrm{mg} / \mathrm{semana})$

\begin{tabular}{lcc}
\hline Ácidos grasos & $\begin{array}{c}\text { Intra-útero } \\
\mathbf{( 2 6 - 4 1} \\
\text { semanas) }\end{array}$ & $\begin{array}{c}\text { Extra-útero } \\
\mathbf{( 0 - 1 0} \\
\text { semanas) }\end{array}$ \\
\hline Omega-9 & 31.2 & 65.5 \\
Omega-6 & 32.8 & 82.4 \\
Omega-3 & 14.6 & 5.5 \\
\hline
\end{tabular}

Fuente: Modificado de Valenzuela et al., 2001.

Estudios electrofisiológicos y conductuales en roedores deficientes en ácidos grasos esenciales, mostraron efectos conductuales atribuibles a una acumulación más baja de lo comúnmente encontrado de DHA y AA en el cerebro (Marmor et al., 2004; Cheatham et al., 2006). Otros estudios del mismo tipo han sido realizados en primates y humanos (Marmor et al., 2004; Cheatham et al., 2006). Un estudio en niños de bajo peso al nacer, después de 7 años de seguimiento, mostró una diferencia en el coeficiente intelectual entre niños que recibieron de su madre la leche humana que entre aquellos que recibieron fórmula conteniendo sólo el precursor del AA (Agostini et al., 1995). Mientras tanto, en niños a término la evidencia es aún menos concluyente, y parece existir un consenso de que el niño es perfectamente capaz de sintetizar tanto el DHA como el AA en suficiente cantidad para el desarrollo óptimo del cerebro, si la dieta contiene precursores de las dos series en proporciones adecuadas (Agostini et al., 1995; Bouwstra et al., 2005; Cheatham et al., 2006).

En cuanto al desarrollo visual, estudios en animales y humanos han documentado diversos efectos y concentraciones de los LC-PUFA de la dieta en la función retinal y la visión (Agostini et al., 1995; Cheatham et al., 2006; Mourek y Mourek Jr, 2011). El tejido visual es una estructura derivada del sistema nervioso y que al igual que el cerebro tiene una extraordinaria capacidad para captar DHA desde el plasma, aunque tampoco está claro si también tiene la capacidad para formar DHA a partir de precursores de menor tamaño (Valenzuela y Nieto, 2001). En la retina el DHA forma parte de los fotorreceptores de los conos y bastones (Valenzuela y Nieto, 2001).

Estas estructuras de la membrana, asociadas a la rodopsina, participan en la conversión del estímulo luminoso en un estímulo eléctrico (depolarización de membranas) y en los procesos de transducción de señales que acompañan a este fenómeno (Valenzuela y Nieto, 2001).

No hay evidencias de que la retina pueda sintetizar DHA a partir de sus precursores. Sin embargo, este ácido graso es continuamente reutilizado en el tejido ya que el recambio de los conos y de los bastones es muy activo (Valenzuela y Nieto, 2001). Estas células desprenden continuamente segmentos de la membrana $(10 \%$ de su estructura diariamente), que son fagocitados por las células del epitelio pigmentado de la retina, produciéndose así una activa reutilización de los productos de la fagocitosis, entre ellos del DHA (Valenzuela y Nieto, 2001). El elevado desarrollo visual asociado con incrementos en la ingesta de LC-PUFA, particularmente DHA, provee la evidencia fuerte de la importancia en estos ácidos grados en la nutrición infantil (Valenzuela y Nieto, 2001; Campoy et al., 2010; Makrides et al., 2011; Mourek y Mourek Jr., 2011).

Dentro de la retina el DHA es incorporado primariamente en glicerofosfolípidos estructurales en la membrana celular (Neuringer, 2000; Ros-Berruezo, 2007). EI DHA en humanos representa del 8 al 20\% de los ácidos grasos totales en la retina y el 38 al $92 \%$ de los PUFA en la retina (Rodríguez et al., 2003). Diversos estudios han provisto la evidencia de un "periodo crítico" durante el desarrollo de la retina cuando un inadecuado suministro de DHA en la retina resulta en una disfunción retinal; aunque realmente se conoce poco acerca del mecanismo íntimo a través del cual el déficit de éste ácido graso altera la función retinal; una cosa si es bien conocida y es que el DHA se encuentra en un alta concentración en la retina, además que éstos fosfoglicéridos contienen los ácidos grasos en posición sn-1 y sn-2 del glicerol (Figura 5) (Neuringer, 2000). Por desgracia y por razones obvias las evidencias de estos "periodos críticos" sólo se conocen a través de la experimentación animal; así en ratas recién nacidas alimentadas con una dieta ca-

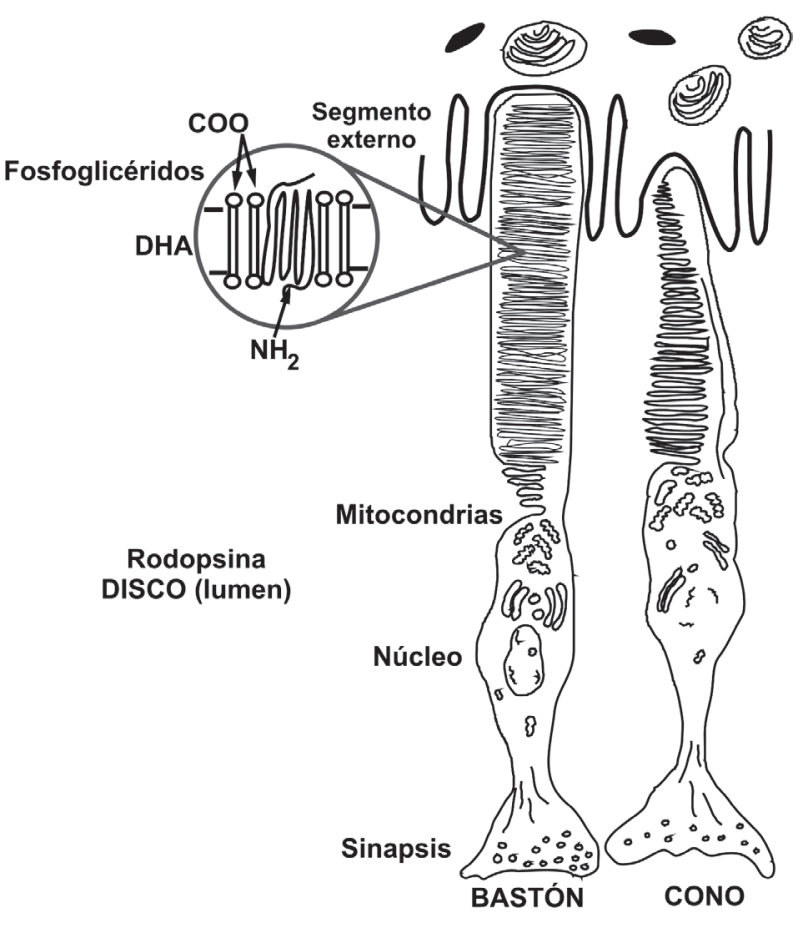

Figura 5

Representación esquemática de conos y bastones y detalle de la membrana de los discos. El segmento externo es el que es capaz de captar los fotones a través de los fotopigmentos. Nótese cómo en la membrana del disco, los fotogliceróles se disponen en empalizada con la cabeza polar hacia el exterior y los ácidos grasos (50\% DHA) orientados hacia el interior. Fuente: Modificado de Neuringer, 2000. 
rente de $\alpha$-LNA y reintroduciéndolo a diversas edades postnatales, en un momento determinado y cuando ésta es tardía, los niveles de DHA se normalizan en la retina, pero no el electrorretinograma (Neuringer, 2000; Ros-Berruezo, 2007). El hecho de que esto ocurriese a las ocho semanas de vida tiene escasa significación para el humano, ya que la rata tiene una retina en la que dominan los bastones y es carente de fóvea en razón de su vida nocturna, que requiere de una buena visión en la oscuridad; por otro lado están los diferentes tiempos madurativos (Neuringer, 2000). Más útil posiblemente sean los estudios realizados, en los que, siguiendo un diseño similar, fueron alimentados con una dieta baja en $\alpha$-LNA durante los 10-20 primeros meses de vida y posteriormente se les cambio a un alimento que contenía DHA $(9 \%)$ durante 9 meses (Neuringer, 2000). Tras este periodo la retina queda depleccionada, pero no se ha normalizado el electrorretinograma. Sin lugar a dudas, estos periodos críticos existen en el niño y especialmente en el pretérmino, en el cual si no hay un aporte adecuado de DHA, la retina sufre una disfunción permanente.

\section{CONCLUSIONES}

La nutrición materna es de vital importancia desde antes de la concepción y durante el embarazo y lactancia. La composición de los ácidos grasos de la leche es un reflejo de los ácidos grasos en la ingesta de la madre, por lo que es necesario que la madre los consuma, algunos estudios han demostrado la asociación de los LC-PUFA con el desarrollo cerebral del feto y del neonato; sobre todo durante el tercer trimestre de embarazo, cuando las demandas fetales para el crecimiento vascular y neuronal son mayores.

Hoy por hoy, es de gran interés el papel que tienen los LC-PUFA, particularmente el omega 3 ácido docosahexaenoico y el omega 6 ácido araquidónico en el crecimiento cerebral que ocurre entre el tercer trimestre del embarazo y el primer año de vida.

En los casos que no es posible suministrar la leche materna al lactante, se tiene la opción de sustituirla por fórmulas lácteas, las cuales deben garantizar ser inocuas y lo más similar posible a la leche humana. Con ello se desprenden resultados positivos en la alimentación y desarrollo del lactante, por ejemplo en algunos estudios se ha encontrado que niños alimentados con fórmulas lácteas enriquecidas con DHA tuvieron una escala IQ verbal superior que niños alimentados con fórmulas sin enriquecer.

\section{REFERENCIAS}

Adamkin D. 2007. Controversias en nutrición neonatal: ácido docosahexanoico (DHA) y nucleótidos. J. Perinatol. 27, S79-S82.
Agostoni C, Trojan S, Bellu R, Riva E, Giovannini M. 1995 Neurodevelopmental Quotient of Healthy Term Infants at 4 Months and Feeding Practice: The Role of Long-Chain Polyunsaturated Fatty Acids. Pediat. Res. 38, 262-266.

Auestad N, Scott D, Janowsky J, Jacobsen C, Carroll R, Montalto M, Halter R, Qu W, Jacobs J, Connor W, Connor S, Taylor J, Neuringer M, Fitzgerald K, Hall R. 2003. Visual Cognitive, and Language Assessments at 39 months. A Follow-up Study of Children Fed Formulas Containing Long-Chain Polyunsaturated Fatty Acids to 1 Year of age. Pediatrics.112, e177-e183.

Bosch V, Golfeno I, Alonso H, Laurentin Z, Materan M, García N. 2009. Ácidos grasos de la leche maternal de mujeres venezolanas de estratos socioeconómicos bajos: Influencia de la temperatura y tiempo de almacenamiento. Arch. Latinoam. Nutr. 59, 61-65.

Bouwstra H, Dijck-brouwer D, Boehm G, Boersma E, Muskiet F, Hadders-Algra M. 2005. Long-chain polyunsaturated fatty acids and neurological developmental outcome at 18 months in healthy term infants. Acta Pediatr. 94, 26-32.

Campoy C, Cabero L, Sanjurjo P, Serra-Majem L, Anadón A, Morán J, Fraga J. 2010. Actualización, recomendaciones y consenso sobre el papel de los ácidos grasos poliinsaturados de cadena larga en la gestación, lactancia y primer año de vida. Med. Clin. 135, 75-82.

Carmona M. 2005. Lípidos en la nutrición del neonato prematuro. PAC Neonatología 2.Libro6, 416-424.

Carver J. 2003. Advances in nutritional modification of infant formulas. Am. J. Clin. Nutr. 77, 1550-1554.

Cheatham C, Colombo J, Carlson S. 2006. N-3 Fatty acids and cognitive and visual acuity development: methodologic and conceptual considerations. Am. J. Clin. Nutr. 83, 1458-1466.

Colombo J, Kannass K, Jill D, Kundurthi S, Maikranz J, Anderson C, Blaga O, Carlson S. 2004. Maternal DHA and the Development of Attention in Infancy and Toddlerhood. Child Development. 75, 1254-1267.

Contreras A, Herrera Y, Rodríguez L, Pizzarro T, Atalah E. 2011. Acceptability and consumption of a dairy drink with omega-3 in pregnant and lactating women of the national supplementary food program. Rev. Chil. Nutr. 38, 313-320.

Coronado M, Vega y León S, Gutiérrez R, García B, Díaz G. 2006. Los Ácidos grasos Omega-3 y Omega-6: Nutrición, Bioquímica y Salud. REB. 25, 72-79.

Cunninghaim P, McDermott L. 2008. Long Chain PUFA Transport in Human Term Placenta. J. Nutrit. 139, 636-639.

Eritsland J. 2000.Safety considerations of polyunsaturated fatty acids. Am. J. Clin. Nutr. 71, 197S-201S.

Fernández J. 2010. Ácidos grasos omega-3 y prevención cardiovascular. Rev. CENIC. 41, 3-15.

García E, Gutiérrez S, Nolasco H. 2007. Desarrollo de bebidas lácteas enriquecidas con ácidos grasos. Rev. Tecnol. Láctea Latinoam. 36-online. www.publitec.com

García R. 2011. Composición e inmunología de la leche humana. Acta Pediatr. Mex. 32, 223-230.

Georgieff M. 2007. Nutrition and the developing brain: nutrient priorities and measurement. Am. J. Clin. Nutr. 85, 614S-220S.

Gil A, Uauy R, Dalmau J. 2006. Comité de Nutrición de la AEP. Bases para una alimentación complementaria adecuada de los lactantes y los niños de corta edad. An. Pediatr. 65, 481-495. 
Gil-Campos M y Dalmau J. 2010. Importancia del ácido docosahexaenoico (DHA): funciones y recomendaciones para su ingesta en la infancia. An. Pediatr. 73, 142.e1.-142.e8.

Haschke-Becher E, Fazzolari-Noesci A, Minoli I, Räihä N, Carrié A-L, Haschke F, Bachman C. 2001.Perfiles de ácidos grasos en lactante alimentados con formulas suplementadas con ácidos grasos poliinsaturados de cadena larga. Nestle Nutrition Workshop Series. Pediatric Program. 47(S), 49-53.

Innis S. 2003. Perinatal biochemistry and physiology of long-chain polyunsaturated fatty acids. J. Pediatr. 143, S1-S8.

Innis S. 2007. Metabolismo lipídico del lactante prematuro. Pediatr. Rev. 24, 256-265.

Koletzko B, Agostoni C, Carlson SE, Clandinin T, Hornstra G, Neuringer M, Uuay R, Yamashiro Y, Willatts M. 2001. Long chain polyunsaturated fatty acids (LCPUFA) and perinatal development. Acta Pediatr. 90, 460-464.

Lama R, Moráis A. 2005. Las grasas en la alimentación infantil. Importancia de los ácidos grasos poliinsaturados. An. Pediatr. Monogr. 3,16-23.

Lindquist S, Hernell O. 2010. Lipid digestion and absorption in early life: an update. Curr. Opin. Clin. Nutr. 13, 314-320.

Macías S, Rodríguez S, Ronayne de Ferrer P. 2006. Leche materna: composición y factores condicionantes de la lactancia. Arch. Argent. Pediatr. 104, 423-430.

Makrides M, Collins C, Gibson R. 2011. Impact of fatty acid status on growth and neurobehavioural development in humans. Matern. Child Nutri. 7, 80-88.

Marmor M, Holder G, Seeliger M, Yamamoto S. 2004. Standard for clinical electroretinography (2004 update). Doc. Opthalmol. 108, 107-114

Martínez V, Aranceta J, Dalmau J, Gil A, Lama R, Martín M, Moreno J, Pavón P, Suárez L. 2009. Recomendaciones nutricionales en la infancia. JANO. 1749, 42-47.

Martinez A y Ballew M. 2011. Infant formulas. Pediatr. Rev. 32, 179-189.

Mc Namara R, Carlson S. 2006. Role of omega-3 fatty acids in brain development and function: Potential implications for the pathogenesis and prevention of psychopathology. Prostag. Leukotr. Ess. 75, 329-349.

McGregor J, Carlson S, Hobel C, Mei S, Ogburn P. 2003. Nutritional roles of omega-3 fatty acids during pregnancy and neonatal development. OBG Management. DowdenHealth Media Inc. (Suppl), S1-S12.

Mena P, Milad A. 1998. Variaciones en la composición nutricional de la leche materna. Algunos aspectos de importancia clínica. Rev. Chil. Pediatr. 69, 116-121.

Mepham T. 1993."Humanizing" milk: The formulation of artificial feeds for infants (1850-1910). Med. His. 37, 225-249.

Minihane A. 2009. Nutrient gene interactions in lipid metabolism. Curr. Opin. Clin. Nutrit. 12, 357-363.

Morales E. 1994. Ácidos grasos poliinsaturados de cadena larga en la nutrición del lactante. Rev. Hosp. Mat. Inf. Ramón Sardá. 13, 73-75.

Morris G, Moorcraft J, Mountjoy A, Wells JCK. 2000. A novel infant formula milk with added long-chain polyunsaturated fatty acids from single-cel sources: a study of growth, satisfaction and health. Eur. J. Clin. Nutrit. 54, 883-886.

Mourek J, Mourek J Jr. 2011. Developmentally dependent and different roles of fatty acids omega- 6 and omega-3. Prague Medical Report. 112, 81-92.

Neuringer M. 2000. Infant vision and retinal functi9on in studies of dietary long-chain polyunsaturated fatty acids: methods, results, and implications. Am. J. Clin. Nutr. 71, 256S-267S

Novo L. 2010. Los lípidos en la nutrición infantil. Rev. Cubana Aliment. Nutr. 20, S1-S28.

Paulou A, Badiola J, Anadón A, Bosch A, Cacho J, Cameán $A$, Cepeda $A$, Domínguez L, Farré $R$, Juárez $M$, Martín F, Martín M, Mas A, Ortega T, Otero A, Paseiro $P$, Ramón D, Rodríguez E, Vidal M, Zurera G. 2010. Informe del Comité Científico de la Agencia Española de Seguridad Alimentaria y Nutrición (AESAN) sobre el riesgo asociado a la presencia de ácidos grasos trans en alimentos. Revista del Comité Científico.12, 95-114.

Prosser C, Svetashev V, Vyssotski M, Lowry D. 2010. Composition and distribution of fatty acids in triglycerides from goat infant formulas with milk fat. J. Dairy Sci. 93, 2857-2862.

Rodriguez A, Raederstorff D, Sarda P, Lauret C, Mendy F, Descomps B. 2003. Preterm infant formula supplementation with $\alpha$ linolenic acid and docosahexaenoic acid. Eur. J. Clin. Nutrit. 57, 727-734.

Rodríguez Y, Pita G, Martín I, Ferret A, Puentes I. 2004. Las grasas en la dieta materna, edad gestacional y peso al nacer. Rev. Cubana Salud Pública. 30, 1-10.

Ros-Berruezo G. 2007. Importancia del componente graso en la dieta del niño de 6 a 12 meses de edad. Anal. Real Acad. Ciencias Veterinarias de Andalucía Oriental. 20, 69-90.

Rosenfeld E, Beyerlein A, Hadders-Algra M, Kennedy K, Singhal A, Fewtrell M, Lucas A, Koletzko B, Von Kies R. 2009. IPD meta-analysis shows no effect of LCPUFA supplementation on infant growth at 18 months. Acta Pediatr. 98, 91-97.

Sala-Vila A, Castellote A, Campoy C, Rivero M, Rodríguez-Palmero M, López-Sabater C. 2004. The source of Long-Chain PUFA in Formula Supplements Does Not Affect the Fatty Acid Composition of Plasma Lipids in Full-Term Infants. J. Nutr. 134, 868-873.

Sánchez C, Narciso D, Rivero M, Sánchez S, Johnston S, Sánchez J, Rodríguez A, Cubero J. 2008. Nociones en alimentación y nutrición infantil durante el primer año de vida. Enfermería Global. 12, 1-8.

Sanhueza J, Nieto S, Valenzuela A. 2004. Ácido docosahexaenoico (DHA), el desarrollo cerebral, memoria y aprendizaje: La importancia de la suplementación perinatal. Rev. Chil. Nutr. 31, 1-15.

Sanjurjo, Trebolazabala N, Aldámiz-Echevarría L, Castaño L, Prieto J, Lodeiro A. 2008. Ácidos grasos n-3 y n-6 en plasma al nacer y al año de edad y relación con el tipo de alimentación. An. Pediatr. 68, 570-575.

Simopoulos A. 1989. Summary of the NATO Advance3d Research Workshop on Dietary $\omega$. and $\omega 6$ Fatty Acids: Biological Effects and Nutritional Essentiality. J. Nutr. 119, 521-528.

Surh J, Lee S, Kwpn H. 2007. 4-Hydroxy-2-alkenal in polyunsaturated fatty acids-fortified infant formulas and other commercial food products. Food Addit. Contam. 24, 1209-1218.

Uauy R, Dangour A. 2009. Fat and fatty acid requirements and recommendations for infants of $0-2$ years and children of 2-18 years. Ann. Nutr. Metab. 76-96.

Valenzuela A, Nieto S. 2001. Ácido dosahexaenoico (DHA) en el desarrollo fetal y en la nutrición maternoinfantil. Rev. Med. Chil. 129, 1-14

Valenzuela A, Nieto S. 2003. Ácidos grasos omega-6 y omega-3 en la nutrición perinatal: su importancia en el desarrollo del sistema nervioso y visual. Rev. Chil. Pediatr. 74, 149-157. 
Valenzuela A, Nieto S, Sanhueza J, Morgado N, Rojas I, Zañartu P. 2010. Supplementing female rats with DHA-Iysophosphatidylcholine increases docosahexaenoicacido and acetylcholine contents in the brain and improves the memory and learning capabilities of the pups. Grasas Aceites. 61, 16-23.

Valenzuela R, Tapia G, González M, Valenzuela A. 2011. Omega-3 fatty acids (EPA and DHA) and its application in diverse clinical situations. Rev. Chil. Pediatr. 38, 356-367.

Vásquez E. 2005. Sucedáneos de la leche materna. SAM Nutrición Pediátrica. Libro 1. Alimentación en el primer Año de Vida. Editores Intersistemas.
Vásquez-Garibay E, Romero-Velarde E. 2008. Esquemas de alimentación saludable en niños durante sus deferentes etapas de la vida. Parte 1. Primeros dos años de vida. Bol. Med. Hosp. Infant. Mex. 65, 593604.

Whelan J. 2008. (n-6) n (n-3) Polyunsaturated Fatty acids and the aging Brain: food for thought. J. Nutr. 138, 2521-2522.

Recibido: $11 / 8 / 11$ Aceptado: 15/11/11 